\title{
Using the Pelvic Floor Impact Questionnaire and Life- style Modifications to Improve Symptoms of Pelvic Floor Dysfunction
}

\author{
Marc Bonis $^{* 1}$, Jenn Lormand ${ }^{2}$ and Christina Walsh ${ }^{3}$ \\ ${ }^{1}$ Assistant Professor, Human Performance and Health Promotion Program, USA \\ ${ }^{2}$ Clinical Exercise Physiologist/ Researcher, Human Performance and Health Promotion Program, USA \\ ${ }^{3}$ Licensed Physical Therapist/ Researcher, Human Performance and Health Promotion Program, USA
}

*Corresponding author: Marc Bonis, Assistant Professor, Human Performance and Health Promotion Program, New Orleans, Louisiana, 2000 Lakeshore Drive ELCF Dept 348K, New Orleans, USA

\section{ARTICLE INFO}

Received: 蔧 April 24, 2019

Published: 幽 April 30, 2019

Citation: Marc Bonis, Jenn Lormand, Christina Walsh. Using the Pelvic Floor Impact Questionnaire and Lifestyle Modifications to Improve Symptoms of Pelvic Floor Dysfunction Biomed J Sci \& Tech Res 17(4)-2019. BJSTR. MS.ID.003029.

Keywords: Pelvic Floor Dysfunction; Pelvic Floor Disorder; Pelvic Floor Muscles

Abbreviations: PFD: Pelvic Floor Disorders; PFM: Pelvic Floor Muscles; IRB: Institutional Review Board

\section{ABSTRACT}

Objective: The purpose of the study was to determine if lifestyle modifications along with a specialized exercise program improved symptoms and pain levels due to pelvic floor dysfunction.

Subjects: 36 women aged $45.5+9.1$ years old (mean + s.d.) identified with symptoms of pelvic floor dysfunction.

Methods: The subjects were assessed for symptoms of pelvic floor dysfunction using the Pelvic Floor Impact Questionnaire (PFIQ-7). After a 30-day control period of no changes to lifestyle and activities, the subjects were introduced to lifestyle modifications and a specialized exercise program that included eight 45-minute live group sessions and a home exercise program to be performed at least three times per week. The subjects were re-assessed after completion of the intervention. IBM SPSS Version 24 statistical programming was used, and Wilcoxon non-parametric analyses were applied to compare the pre- and post-intervention data to determine if significant relief from dysfunction occurred.

Results: The intervention significantly improved pelvic floor disorder symptoms (PFIQ-7, $\mathrm{z}(36)=-3.777, \mathrm{p}<0.05)$ and diastasis recti separation, $(\mathrm{z}(36)=-4.926, \mathrm{p}<0.05)$. The level of significance was set at $\mathrm{p}=0.05$.

Conclusion: Lifestyle modifications and a specialized exercise program can significantly improve symptoms of pelvic floor dysfunction.

\section{Introduction}

Pelvic floor disorders (PFD) occur when the muscles that comprise the pelvic floor fail to properly contract. This can cause urinary incontinence, pelvic organ prolapses, fecal incontinence, or other sensory and emptying abnormalities of the lower urinary and GI tracts [1,2]. Current evidence shows that individuals with low back pain have a significant decrease in pelvic floor function compared to individuals without LBP [3]. Over $25 \%$ of all women and more than a third over the age of 65 experience PFD. Even though it is a physiological problem, the psychosocial impact of PFD can be much more detrimental to the patient's quality of life. Over the next 30 years chronic health problems associated with PFD are projected to increase by $50 \%$ due to the increasing numbers of women reaching age 65 [4]. PFD does not typically have one specific cause. Pregnancy/childbirth, age, hormonal changes, obesity, lower UTI, and pelvic surgery are major risk factors. Additionally, anatomical, physiological, genetic, reproductive and lifestyle components are probably PFD developmental influences $[1,4]$. 
The pelvic floor forms the inferior border of the abdominopelvic cavity [4]. It supports the abdomino-pelvic organs. The pelvic floor muscles (PFM) function as a unit instead of individually contracting. They play an important role in maintaining and increasing intra-abdominal pressure during functional tasks such as lifting, sneezing, coughing, and laughing to prevent urinary and fecal incontinence [3,5]. Men can also have disorders of the pelvic floor, however due to the anatomy of the male pelvis, it is less common [6]. Current evidence supports exercise protocols with the common goal of regaining neuromuscular control of the pelvic floor and deep abdominal muscles in a functional matter [7]. There is also strong evidence for PFM training as conservative treatment for stress urinary incontinence $[5,8]$. Treatment should also include education on healthy lifestyle habits to promote optimal functioning of the lumbopelvic stability system. Examples of these habits include good posture, maintenance of a healthy body weight, proper diet, routine exercise, and refraining from smoking [6]. The purpose of this study is to test the hypothesis that specific lifestyle and exercise interventions will offer statistically significant improvements in symptoms of pelvic floor dysfunction in women.

Table 1: Subjects' Physical Characteristics.

\section{Methods}

The current research evaluated whether lifestyle modifications as well as a specialized exercise program improved the symptoms of pelvic floor dysfunction in women. These symptoms include low back pain, hip pain, pelvic pressure, pelvic pain with intimacy and/ or the use of tampons, bladder and/or bowel leakage with laughing, coughing, sneezing, jumping, bladder and/or bowel urgency and frequency. The study was approved by the Institutional Review Board (IRB) of the University of New Orleans.

\section{Subjects}

The study consisted of 36 female subjects between the ages of 28-69 years old. The subjects were recruited as a convenience sample from the Greater New Orleans area through the use of informational fliers, Facebook video ads, e-mail messages of the same flier, and community postings of the flier. Prior to participation in the study the subjects signed a letter of informed consent stating that the study was voluntary and confidential and that all results would be kept in a locked, secure environment. No remuneration was provided to the subjects. During the course of the study one participant was dropped from the research due to non-compliance. See Table 1 for the participants' physical characteristics.

\begin{tabular}{|c|c|c|c|c|c|}
\hline \multicolumn{7}{|c|}{ Subjects' Physical Characteristics } \\
\hline $\mathbf{n}$ & Gender & Age (yr.) (mean + s.d.) & Height (m) (mean + s.d.) & Weight (kg) (mean + s.d.) & BMI (mean + s.d.) \\
\hline 36 & Female & $45.9+9.1 \mathrm{yrs}$ & $1.59+.1 \mathrm{~m}$ & $69.2+12.3 \mathrm{~kg}$ & $25.8+3.2$. \\
\hline
\end{tabular}

\section{Participation Criteria}

Research inclusion consisted of women that exhibited symptoms of pelvic floor dysfunction as defined by the Pelvic Floor Impact Questionnaire - short form 7 (PFIQ-7) [9]. Subjects were excluded from the study if they were assessed to have greater than a 3 finger-width diastasis recti abdominal separation as measured by one of the co-investigators. Exclusion criteria ensured that those with advanced symptoms were referred for medical consultation. All participants had ample opportunity for questions to be answered as needed by the co-investigator clinicians during the live sessions.

\section{Design}

The participants were assessed three times. All tests and assessments listed below were administered pre and post control period and post intervention period.

a) The Pelvic Floor Impact Questionnaire: Short form 7 (PFIQ-7) measured how much bladder, bowel, or vaginal symptoms affected the participant's activities, relationships, and feelings $[9,10]$. Scores on this questionnaire were used to determine if statistically significant improvements in symptoms occurred for the participants:

b) A Diastasis Recti assessment measured any separation of the abdominal muscles that may be present, and if applicable the extent to which it is present [14]. The participant lay supine on the examination table and lifted her head from the table while one of the co-investigators applied gentle pressure with 1-3 fingers along the center-line of the abdomen. Scoring was as follows:

1. 0 - no separation present

2. $0.5-1 / 2$ finger-width separation

3. 1 - 1 finger-width separation

4. $\quad 1.5$ - 1.5 finger-width separation

5. 2 - 2 finger-width separation

6. $2.5-2.5$ finger-width separation

7. 3 - 3 finger-width separation

\section{Procedure}

The participants were assessed to determine their level of pain and dysfunction. A 30-day control period was established to determine baseline data for a comparison to the results of the study's intervention. The participants were re-assessed after the 30-day control period without any change to their current activities and routines to determine the chronic nature of their pain, and/or dysfunction and to record the subjects' pre-intervention levels. The participants were then introduced to lifestyle modifications and were taught an exercise program over the course of eight live group 
sessions, 45 minutes each, led by one or both of the co-investigators. One or both of the co-investigators provided verbal instruction as well as demonstrations and tactile assistance as needed to ensure safe and correct performance of the lifestyle interventions and exercises.

Participants were also required to perform a home exercise program at least 3 additional days per week. Finally, the participants were re-assessed a third time after the intervention was completed to determine if there were any changes in pain and/or dysfunction.

The two research clinicians were a licensed Physical Therapist and a clinical exercise physiologist who administered the entire protocol to all of the participants. The third member of the research group was an experienced researcher and faculty member from the University of New Orleans who participated as the primary investigator and methodologist for the study.

\section{Specialized Exercise Program and Lifestyle Modifications}

The co-clinician investigators began the intervention period with a brief pelvic anatomy lesson, as well as instruction in diaphragmatic breath-work, low back decompression, and the proper use of the Muscletrac roller for myofascial release. The subjects were introduced to lifestyle modifications such as: the proper use of a Squatty Potty for bowel and bladder elimination, proper hydration and fiber consumption, avoiding compression garments, and avoiding high-heeled shoes. They were also instructed in exercise phases using the Pelvicore which consisted of tri-planar activation exercises performed with both abduction and adduction of the hips. The 3 phases of exercise instruction included a standing series, plank series, and bridge series of exercises. This unique exercise protocol functionally activates and integrates all of the foundational strength muscles of the core and pelvis, specifically the abdominals (especially transverse abdominis), hip abductors (gluteus medius), hip adductors, pelvic floor muscles, back extensors (multifidus), and glutes (gluteus maximus). The participants were also provided with on-demand access to videos of the co-investigator clinicians teaching / reviewing the exercises.

\section{Limitations}

The 3 additional-day per week home exercise portion of the protocol was self-administered and self-reported by the study's participants.

\section{Analysis}

IBM SPSS Version 24 statistical programming was used and non-parametric Wilcoxon "related-items" analyses were employed [11] for both the pre- and post-control period and the pre- and post-protocol period. A 0.05 level of significance was used.

\section{Results}

Comparisons between pre- and post-control period data indicated no significant differences in the participants' pelvic floor quality of life (PFIQ-7, z (36) $=-.886, \mathrm{p}>0.05$ ) and diastasis recti differences $(z(36)=-.83, p>0.05)$. See Table 2 for the results of the control period comparisons. Comparisons between pre- and postprotocol data indicated significant differences in the participants' pelvic floor quality of life (PFIQ-7, z (36) = -3.832, p < 0.05) and diastasis recti differences $(z(36)=-4.719, p<0.05)$. See Table 3 for the results of the protocol comparisons.

Table 2: "Pre and Post" Control Period Comparisons.

\begin{tabular}{|c|c|c|}
\hline \multicolumn{3}{|c|}{ "Pre and Post" Control Period Comparisons } \\
\hline Assessment & $z(36)$ & Significance * \\
\hline PFIQ-7 Quality of Life Composite & -.886 & .323 \\
\hline PFIQ-7 (Bladder Component) & -.591 & .541 \\
\hline PFIQ-7 (Bowel Component) & -.088 & .876 \\
\hline PFIQ-7 (Prolapse Component) & -.561 & .547 \\
\hline Diastasis Recti Separation & -.830 & .392 \\
\hline \multicolumn{3}{|c|}{ *Level of Significance $p=0.05$} \\
\hline
\end{tabular}

Table 3: "Pre and Post" Intervention Comparisons.

\begin{tabular}{|c|c|c|}
\hline \multicolumn{3}{|c|}{ "Pre and Post" Intervention Comparisons } \\
\hline Assessment & $z(36)$ & Significance * \\
\hline PFIQ-7 Quality of Life Composite & -3.832 & $.000 *$ \\
\hline PFIQ-7 (Bladder Component) & -3.666 & $.000 *$ \\
\hline PFIQ-7 (Bowel Component) & -2.314 & $.032 *$ \\
\hline PFIQ-7 (Prolapse Component) & -3.602 & $.011^{*}$ \\
\hline Diastasis Recti Separation & -4.719 & $.001^{*}$ \\
\hline \multicolumn{3}{|c|}{${ }^{*}$ Level of Significance $\mathrm{p}=0.05$} \\
\hline
\end{tabular}

\section{Discussion}

The intervention significantly improved pelvic quality of life and diastasis recti separation. The women who participated in this research included women who had recently become mothers, those whose youngest children were teenagers, as well as a few women who were diagnosed with a lax connective tissue disorder (Ehlers-Danlos Syndrome). All of them benefited from statistically significant symptom relief. Women dealing with symptoms that were successfully treated using this intervention often seek help and relief from many different physicians and practitioners, or don't know where to turn for help. Prior to the current research there has not been a highly effective option to re-activate the necessary core muscles to relieve the symptoms.

This intervention was effective because it strengthened the foundational muscles of the body - the core and re-activated deep support muscles of the core that have often lain dormant since a precipitating incident, including most often pregnancy and childbirth, or simply due to disuse over many years possibly as a result of a seated occupation or sedentary lifestyle. Previous interventions focused on strengthening these muscles from an isometric / bracing perspective which does not result in functional strength or support in movement.

\section{Conclusion}

Further research using the current intervention is recommended to address lumbopelvic distress associated with lower back pain, pelvic floor dysfunction, and pelvic organ prolapse. 
The implications of applying the success of the current research to an expanded area of research is far-reaching, as the size of the population suffering from lumbopelvic symptoms includes at least $1 / 3$ of all women. In order to insure the successful application of future research the current intervention would be expanded into a protocol that improves both the assessment and therapeutic aspects of the current intervention.

In addition to using assessment tools of the Pelvic Floor Impact Questionnaire (PFIQ-7) and diastasis recti separation, the protocol would include additional assessment tools, such as the Oswestry Low Back Pain Questionnaire [10,12] and the Pelvic Floor Distress Inventory (PFDI-20) $[9,10]$. Additional therapeutic aspects would include pelvic alignment [13], pelvic floor muscle activation [14], and InBody570 body composition measurement [15]. A powerful potential use of this protocol would be in the earlier intervention phase in the course of the lumbopelvic symptoms noted. The savings in medical expense over the subjects' lifespan as well as in mental and emotional strain could mount to phenomenal proportions as there is great potential for back, pelvic floor, and organ surgical procedures to be avoided.

\section{Conflict of Interest}

No conflict of interest exists for this study.

\section{References}

1. Eliasson K, Elfving B, Nordgren B, Mattsson E (2008) Urinary incontinence in women with low back pain. Manual Therapy 13(3): 206212.

2. Nygaard I, Barber M, Brody D, Kenton K, Meikle S, etal. (2008) Prevalence of symptomatic pelvic floor disorders in US women. JAMA: Journal of the American Medical Association 300(11): 1311-1316.

\section{ISSN: 2574-1241}

DOI: 10.26717/BJSTR.2019.17.003029

Marc Bonis. Biomed J Sci \& Tech Res

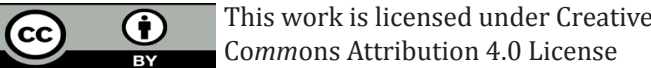

Submission Link: https://biomedres.us/submit-manuscript.php
3. Arab A, Behbahani R, Lorestani L, Azari A (2010) Assessment of pelvic floor muscle function in women with and without low back pain using transabdominal ultrasound. Man Ther 15(3): 235-239.

4. Davis K (2013) Pelvic floor dysfunction causes and assessment. Practice Nursing 21(7): 340-346.

5. Mohseni Bandpei M, Rahmani N, Behtash H, Karimloo M (2011) The effect of pelvic floor muscle exercise on women with chronic nonspecific low back pain. Journal of Bodywork \& Movement Therapies 15(1): 75-81.

6. Christie C, Colosi R (2009) Paving the way for a healthy pelvic floor. IDEA Fitness Journal 6(5): 42-49.

7. O Sullivan P, Beales D (2007) Changes in pelvic floor and diaphragm kinematics and respiratory patterns in subjects with sacroiliac joint pain following a motor learning intervention: a case series. Manual Therapy 12(3): 209-218.

8. Grewar H, McLean L (2008) The integrated continence system: a manual therapy approach to the treatment of stress urinary incontinence. Manual Therapy 13(5): 375-386.

9. Barber M, Chen Z, Lukacz E (2011) Further Validation of the Short Form Versions of the Pelvic Floor Distress Inventory (PFDI) and Pelvic Floor Impact Questionnaire (PFIQ). Neurourol Urodyn 30(4): 541-546.

10. Davidson M, Keating J (2002) A comparison of five low back disability questionnaires: reliability and responsiveness. Phys Ther 82(1): 8-24.

11. Jamieson S (2004) Likert scales: how to (ab)use. Med Educ 38(12): 1217-1218.

12. Fairbank J, Pynsent P (2000) The Oswestry Disability Index. Spine 25(22): 2940-2953.

13. Hall C, Brody L (2005) Therapeutic Exercise: Moving Toward Function. Lippincott Williams and Wilkins, 2nd Edition, display 19-12: Externally Palpating the Pelvic Floor Muscles.

14. Sapsford R, Hodges P (2001) Contraction of the pelvic floor muscles during abdominal maneuvers. Arch Phys Med Rehabil 82(8): 1081-1088.

15. Karelis A, Chamberland G, Aubertin Leheudre M, Duval C (2013) Validation of a portable bioelectrical impedance analyzer for the assessment of body composition. Appl Physiol Nutr Metab 38(1): 27-32.

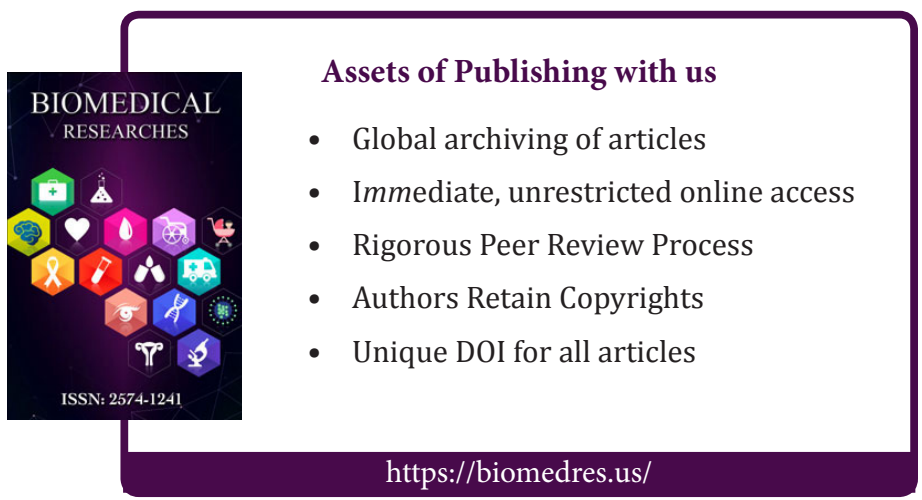

